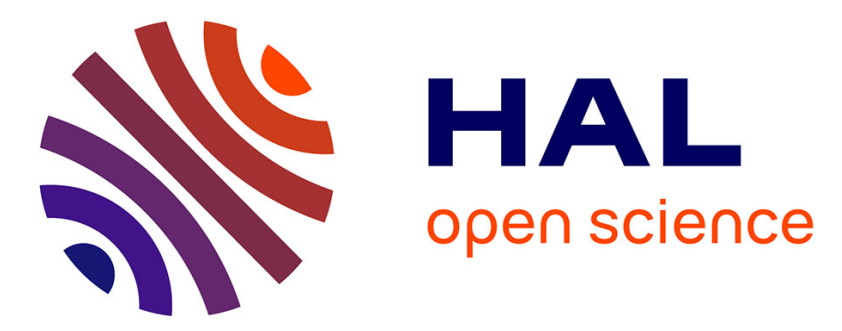

\title{
VARIATIONS DE L'ÉNERGIE RELATIVE DISSIPÉE AU COURS DE LA FATIGUE D'UN ALUMINIUM 5N
}

J. Chicois, C. Esnouf, Gilbert Fantozzi, A. Vincent, R. Fougères

\section{To cite this version:}

J. Chicois, C. Esnouf, Gilbert Fantozzi, A. Vincent, R. Fougères. VARIATIONS DE L'ÉNERGIE RELATIVE DISSIPÉE AU COURS DE LA FATIGUE D'UN ALUMINIUM 5N. Journal de Physique Colloques, 1983, 44 (C9), pp.C9-777-C9-784. 10.1051/jphyscol:19839118 . jpa-00223353

\section{HAL Id: jpa-00223353 https://hal.science/jpa-00223353}

Submitted on 1 Jan 1983

HAL is a multi-disciplinary open access archive for the deposit and dissemination of scientific research documents, whether they are published or not. The documents may come from teaching and research institutions in France or abroad, or from public or private research centers.
L'archive ouverte pluridisciplinaire HAL, est destinée au dépôt et à la diffusion de documents scientifiques de niveau recherche, publiés ou non, émanant des établissements d'enseignement et de recherche français ou étrangers, des laboratoires publics ou privés. 


\title{
VARIATIONS DE L'ÉNERGIE RELATIVE DISSIPÉE AU COURS DE LA FATIGUE D'UN ALUMINIUM $5 \mathrm{~N}$
}

\author{
J. Chicois, C. Esnouf, G. Fantozzi, A. Vincent* et R. Fougères \\ Groupe d'Etudes de Métalzurgie Physique et de Physique des Matériaux', \\ I.N.S.A., 69621 Vilzeurbanne Cedex, France \\ * Laboratoire de Traitement du Signal et d'Ultrasons, I.N.S.A., Bât. 502, \\ 69621 Villeurbanne Cedex, France
}

\begin{abstract}
Résumé - Lors d'essais de fatigue en traction-compression sur Al $5 \mathrm{~N}$ à la température ambiante, nous avons déterminé l'énergie relative dissipée en fonction de différents paramètres : mode de dêroulement du cycle, amplitude et fréquence et sur deux états initiaux différents, recuit et préfatigué à saturation. Les résultats expérimentaux sont analysếs à partir des mécanismes microscopiques proposés habituellement pour expliquer le comportement cyclique.

Abstract- This paper is concerning with the evolution of dissipated relative energy during symmetric push-pull test of $\mathrm{Al} 5 \mathrm{~N}$ at room temperature. On two microstructural state (annealed and prefatigued), effects of cycles conditions, frequency and amplitude strain have been determined. Experimental results have been analyzed as depending on classical microscopic mechanics suggested in fatigue behaviour.
\end{abstract}

\section{INTRODUCTION}

Le comportement des métaux sous contrainte cyclique a fait 1 'objet de nombreuses ëtudes ces dernières annẻes. D'une manière générale, deux types d'approche ont ẻtê développés. Certains auteurs se sont attachés à décrire les microstructures obtenues lors de sollicitations en traction-compression. A partir de ces observations, ils ont tenté de décrire quantitativement 1 'évolution de propriêtés mécaniques. issues de 1 'analyse de cycles contrainte-déformation $(1),(2),(3),(4),(5),(6)$. Les propriétés étudiées sont généralement sensibles aux conditions dans lesquelles sont décrits les cycles (7). Ces études ont généralement été menées sur du cuivre mono ou polycristallin. D'autres auteurs ont cherché à déduire, de mesures de frottement intérieur basse fréquence ou d'atténuation d'ondes ultrasonores, les configurations de dislocations résultant de la déformation cyclique. Ces recherches ont été menées sur des états préalablement fatigués, soit en torsion (8), (9), soit en traction-compression (10).

L'objectif de ce travail est d'utiliser ces deux types d'approche, afin d'analyser les mécanismes microscopiques mis en jeu au cours de sollicitations cycliques d'aluminium $5 \mathrm{~N}$ en mesurant, au cours de la fatigue en traction-compression, 1 'énergie dissipée par cycle et son évolution en fonction de plusieurs paramètres et de différents états.

\section{PROCESSUS EXPERIMENTAL}

La sollicitation cyclique a eu lieu sur une machine de fatigue spêcifique, à cinématique entièrement élastique, pilotẻe par ordinateur et permettant de solliciter les éprouvettes en traction-compression sous des amplitudes de déformation relative variant de quelques $10^{-6}$ à $10^{-2}$ ( $J$. Chicois et al. à parầtre). La sollicitation cyclique est réalisée soit à déformation plastique $\varepsilon$ imposée, soit à déformation totale $\varepsilon_{t}$ imposée : dans le premier cas, 1 'asservissement est réalisé à vitesse de déformation totale constante lors du chargement initial et dans les zones de déchargetaboratoire associé au C.N.R.S. 
ments quasi élastiques ; dès que, pour une contrainte donnée, la déformation totale est supérieure de $5 \%$ à la déformation élastique, 1 'asservissement est alors réalisé à vitesse de déformation plastique $\dot{\varepsilon}_{\text {p }}$ constante; dans le second cas, 1 'asservissement est réalisé à vitesse de déformation totale $\dot{\varepsilon}_{t}$ constante sur 1 'ensemble du cycle.

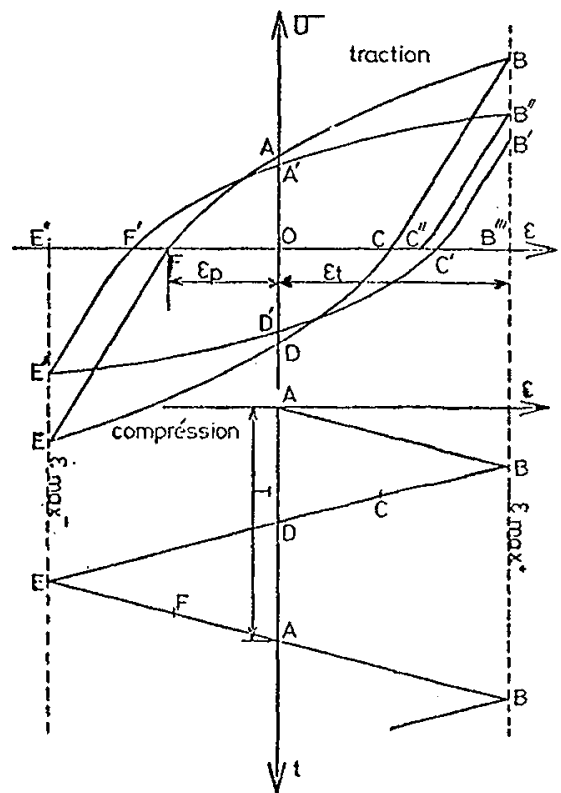

Fig. 1 - Evolution linéaire de la déformation en fonction du temps de sollicitation cyclique. Schéma du cycle de fatigue correspondant dans le cas de la déformation totale imposée.
La figure l représente 1 'évolution Iinéaire de la déformation en fonction du temps entre deux valeurs extrêmes $+\varepsilon$ et $-\varepsilon$, dans le cas des cycles à amplitudes totales imposées.

Le cycle correspondant est schématisé ci-contre, CDEFABC dans le cas d'un cycle fermé, et $C^{\prime} D^{\prime} E^{\prime} F^{\prime} A^{\prime} B^{\prime \prime} C^{\prime \prime}$ dans le cas $d^{\prime}$ un cycle ouvert. L'énergie dissipée relative $\Delta$ est définie par la relation suivante :

$$
\Delta=\frac{\Delta \mathrm{W}}{\mathrm{W}^{+}+\mathrm{W}^{-}},
$$

- $\Delta W$ est I'énergie dissipée par cycle égale à $I^{\prime}$ aire comprise à $1^{\prime}$ intérieur du cycle $C^{\prime} D^{\prime} E^{\prime} F^{\prime} A^{\prime} B^{\prime \prime} C^{\prime \prime}$ (figure 1).

- $W^{+}$et $W^{-}$sont respectivement $I^{\prime}$ aire des triangles OBB" et OEE" :

Nous avons choisi cette définition de 1 'énergie dissipée, car elle correspond à celle du frottement interne $\Delta=\Delta W / 2 W(11)$, lorsque les cycles sont fermés et symêtriques.

Nous avons utilisé deux types d'extensomètres selon l'amplitude de la déformation : pour les faibles amplitudes, quelques $10^{-6}$ à quelques $10^{-4}$,

la déformation de l'éprouvette a été mesurẻe par quatre jauges à fil résistant placées deux par deux sur des faces opposées de l'échantillon. Ces quatre jauges constituent un pont complet permettant d'annihiler les effets de variation de température. Les déformations les plus importantes ont été mesurées par un extensomètre de type Schenck présentant une base de mesure de $20 \mathrm{~mm}$. Dans le domaine de recouvrement des deux extensomètres (de $1^{\prime}$ ordre de quelques $10^{-4}$ ), nous avons vêrifié que les valeurs de 1 'énergie dissipée, calculées à partir des informations données par les deux dispositifs de mesure, étaient identiques.

Tous les essais ont eu lieu à la température ambiante sur des états recuits ou préalablement fatigués. La fréquence des essais était comprise entre $1 \mathrm{~Hz}$ et $1 / 100 \mathrm{~Hz}$.

Nous avons utilisé des éprouvettes de fatigue en aluminium $99,999 \%$ présentant une section carrée de $7 \times 7 \mathrm{~mm}$ et une longueur utile de $50 \mathrm{~mm}$; elles ont été usinées dans des barreaux cylindriques, obtenues par étirage (taux d'écrouissage : $70 \%$ ), puis recuites à une température de $625 \mathrm{~K}$ pendant deux heures.

\section{II - RESULTATS OBTENUS}

\section{- Etats recuits}

Les figures 2 et 3 illustrent, sur des états recuits, 1 'évolution des cycles contraintes-déformations dans les deux conditions de sollicitation retenues. 

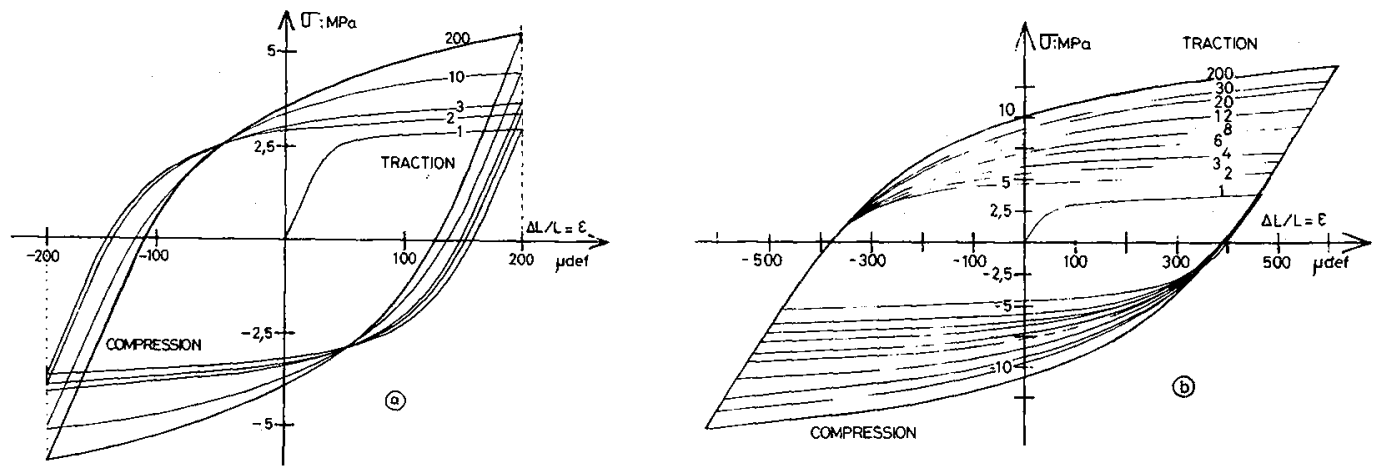

Fig. 2 - Cycles contraintes-déformation à amplitude totale imposée (a) et à amplitude plastique imposée (b), fréquence $1 / 30 \mathrm{~Hz}$.

On remarque un durcissement cyclique dans les deux cas, mais une évolution de la forme des cycles trẽs différente selon que la déformation a lieu à amplitude de déformation totale imposée ou plastique imposée. Ce comportement différent est illustré par la variation de l'énergie relative dissipée en fonction du nombre de cycles (figure 3 ).

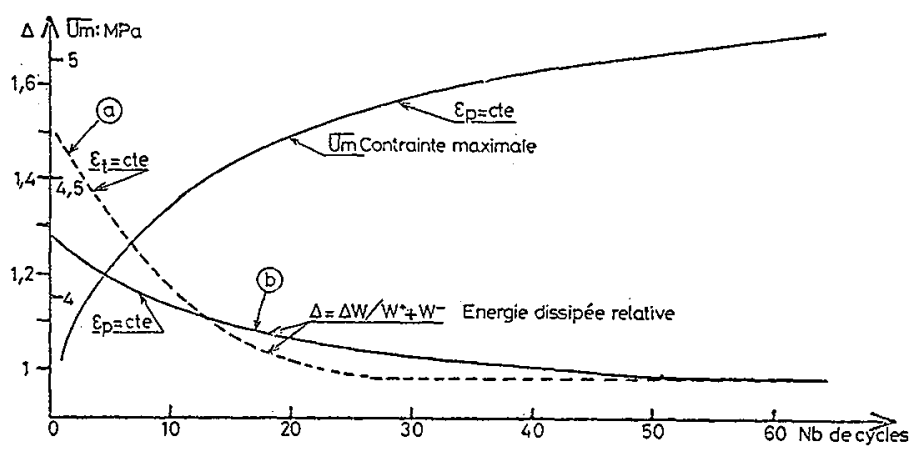

Fig. 3 - Evolution de 1 'énergie relative dissipèe $\Delta=\frac{\Delta W}{W^{+}+W^{-}}$en fonction du nombre de cycles pour une déformation totale à saturation égale à $135.10^{-6}$, fréquence $1 / 30 \mathrm{~Hz}$.

Les courbes a et $b$ de la figure 3 montrent respectivement 1 'évolution de $\Delta=\frac{\Delta W}{W^{+}+W^{-}}$

dans le cas de cycles à amplitude totale imposée et plastique imposée. Ces deux courbes tendent vers une même valeur asymptotique. Nous considérerons que I'état ainsi obtenu est $I^{\prime}$ état saturé. A saturation, I'amplitude totale est la même pour les deux modes de description des cycles. La saturation est atteinte beaucoup plus rapidement lorsque c'est la déformation totale qui est imposée.

La figure 4 représente les évolutions de l'énergie dissipẻe et de la contrainte maximale des cycles (égale à la moyenne des contraintes en traction et en compression lorsqu'il y a une légère dissymêtrie) en fonction du nombre de cycles : ces évolutions sont représentées pour différentes valeurs de 1 'amplitude totale imposée. 


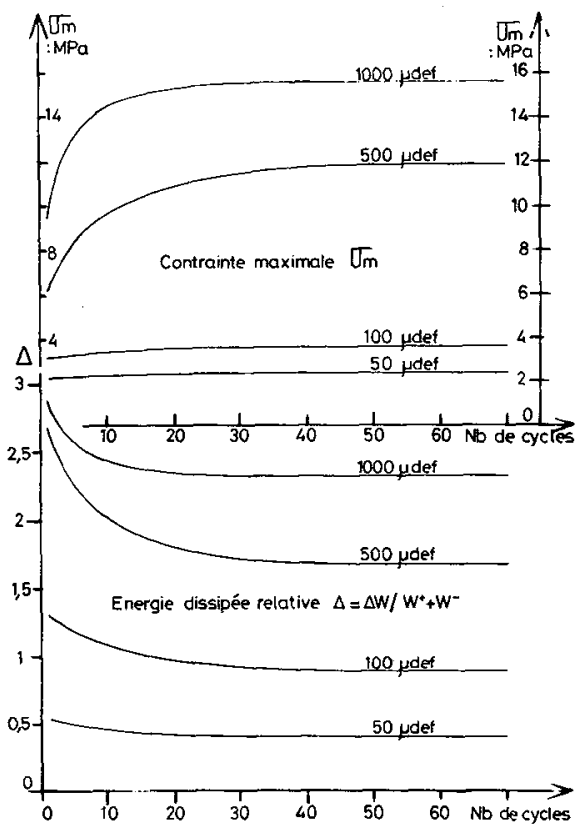

Fig. 4 - Evolution de 1 'énergie relative dissipée et de $1 \mathrm{a}$ contrainte maximale $\sigma_{\mathrm{m}}$ des cycles en fonction du nombre de cycles effectués, fréquence $1 / 30 \mathrm{~Hz}$.

On constate un durcissement régulier en fonction du nombre de cycles : ce durcissement s'accompagne d'une diminution de I'énergie dissipée et d'une augmentation de la contrainte maximale des cycles. L'état saturé est atteint d'autant plus vite que

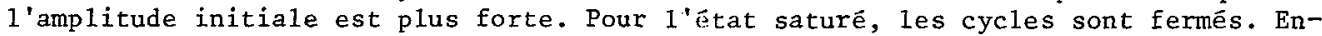
fin, aux basses amplitudes de déformation, la variation relative de $\frac{\Delta W}{+}$ entre le premier cycle et la saturation est plus grande que celle correspondant ${ }^{+} \mathrm{W}_{1}^{+}$la contrainte alors que le phénomène est inverse aux fortes amplitudes. Ce phénomène est bien illustré sur la figure 5, où nous avons reporté les valeurs de contrainte maximale $\sigma_{S}$ et d'énergie dissipée à saturation : Iorsque $\varepsilon$ dépasse $10^{-3}$, on n'observe plus qu'une très faible variation de I'ênergie relative dissipée.

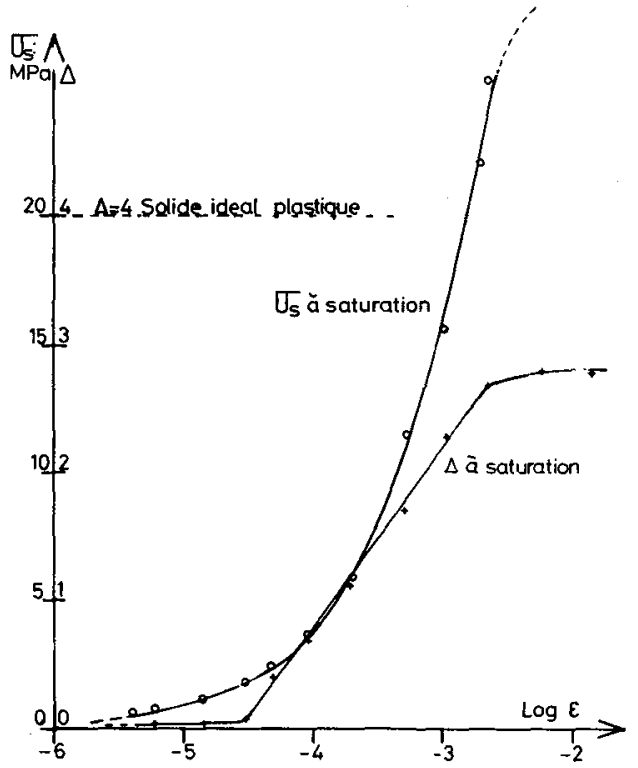

Fig. 5 -Variation de 1 'énergie dissipêe et de la containte maximale des cycles pour I'état saturê en fonction de 1 'amplitude de déformation totale imposée, fréquence $1 / 30 \mathrm{~Hz}$. 
En pointillé, nous avons représenté la valeur de $\frac{\Delta W}{W^{-}+W^{+}}$égale à 4 poux le solide idéal plastique sans consolidation qui constitue une ${ }^{+}$borne supérieure pour les valeurs mesurées.

En-dessous de $10^{-3}$, il existe deux zones distinctes selon 1 'amplitude de déformation (fig. 5) ; en-dessous d'une déformation égale à $3 \cdot 10^{-4}$, on observe une évolution lente de $\Delta$ avec des cycles qui sont pratiquement fermés dès les premiers instants de la sollicitation. De $3 \cdot 10^{-4}$ à $10^{-3}$, 1'augmentation de $\Delta$ est très rapide et les cycles ne deviennent fermés que lorsque la saturation est atteinte.

\section{- Etat recuit + préfatigué}

Sur la figure 6 , nous avons représenté 1 'évolution de 1 'ênergie relative dissipée et de la contrainte maximale en fonction de 1 'amplitude de déformation pour un état recuit et préalablement fatigué, sous une amplitude totale de $2.10^{-3}$. Les valeurs de $\Delta W /\left(W^{+}+W^{-}\right)$sont les moyennes obtenues sur les dix premiers cycles qui sont en général fermés et symétriques. La variation de l'énergie relative dissipée est alors assimilable au frottement intérieur $\Delta=\Delta \mathrm{W} / 2 \mathrm{~W}$. Selon 1 'évolution de $\Delta$ avec 1 'amplitude $\varepsilon_{t}$, on constate trois domaines : $l$ 'un pour les faibles amplitudes de déformation où les variations de $\Delta$ sont lentes, le deuxième tel que $10^{-4}<\varepsilon_{t}<6.10^{-4}$ où l'énergie dissipée crô̂t rapidement et, enfin, un troisième domaine de $6 \cdot 10^{-4}$ à $2 \cdot 10^{-3}$ où 1 'évolution est à nouveau ralentie.

La figure 7 montre $I^{\prime}$ effet de la fréquence $d^{+}$essais lors d'une variation de 1 à $1 / 100 \mathrm{~Hz}$ pour des valeurs de $\varepsilon_{\text {r }}$ correspondant au deuxième domaine dêfini ci-dessus et après saturation à $2 \cdot 10^{-3}$. Entre chaque essai, l'échantillon n'a pas été resollicitéa $2.10^{-3}$. Sur la figure 7 , on peut observer un effet du temps mis pour décrire les cycles. Si, sur un même état initialement saturé, on procède à la séquence d'essais I, $1 / 5,1 / 20,1 / 100 \mathrm{~Hz}$ (séquence 1), puis $1 / 100,1 / 20,1 / 5$ et $1 \mathrm{~Hz}$ (séquence 2), on obtient une des courbes représentées figure 7 . On constate alors une augmentation de $\Delta$ (fig. 7) et une diminution de la contrainte pour la séquence 1 (fig. 6). Par contre, ces grandeurs demeurent constantes pour 1a séquence 2. Cela traduit une légère modification de la microstructure tendant vers un adoucissement pour la séquence 1 et non pas un effet de la fréquence, comme le montrent les résultats de la séquence 2 .

Dans le premier domaine de déformation (fig. 6), on observe une augmentation réversible de $\Delta$ lorsque la fréquence augmente. Le niveau de frottement intérieur est sensible à un vieillissement comme le montre 1'effet d'un chauffage pendant une heure à $365 \mathrm{~K}$ (fig. 6) A $\sigma_{\mathrm{s}}$ :

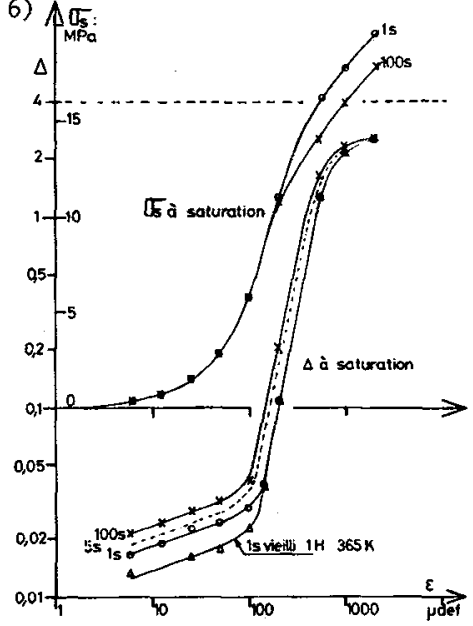

Fig. 6 - Effet de l'amplitude sollicitation sur 1 'énergie dissipée et la contrainte maximale pour un état préfatigué à saturation (amplitude : $2 \cdot 10^{-3}$ ) .

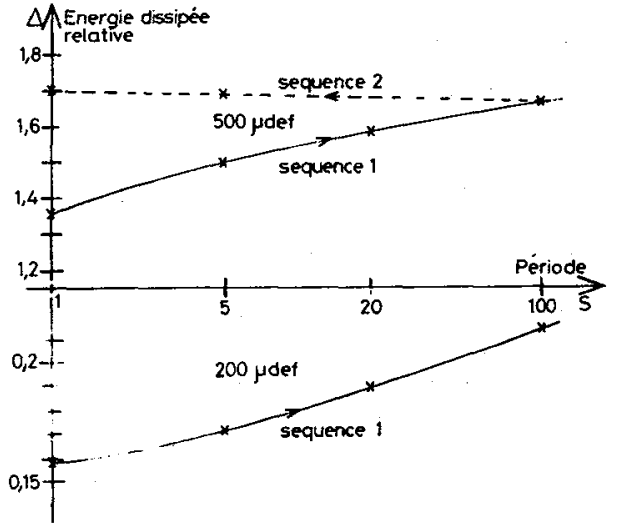

Fig. 7 - Influence du temps de parcours d'un cycle sur 1 'énergie dissipée pour différentes valeurs de déformation. 


\section{III - ANALYSE DES RESULTATS ET INTERPRETATION}

\section{- A 1 'état recuit}

Dans le deuxième domaine, les variations rapides de la contrainte maximale et de 1 'énergie dissipée traduisent un durcissement prononcé correspondant vraisemblablement à une augmentation importante de la densité de défauts introduits. En effet, une étude au MET sur des états fatigués à $6 \cdot 10^{-4}$ d'amplitude totale a mis en évidence une structure cellulaire de dislocations qui apparaît seulement après quelques cycles (Guichon et a1. à parâ̂tre). La taille des cellules est de quelques microns et on observe de plus des dipoles et des bouciles de dislocations. Pour la sollicitation à déformation plastique imposée, dont les résultats sont représentés sur la figure 3, la contrainte maximale $\sigma_{m}$ en fonction du nombre de cycles $N$ suit une loi du type $\sigma_{m^{\alpha N}} l / 2$. Cette relation $n^{\prime} e^{m} t$ valable qu'avant saturation. Si $\varepsilon_{p_{c}}$ est la déformation plastique cumulée $\left(\varepsilon_{\mathrm{pc}}=4 \mathrm{~N} \varepsilon_{\mathrm{p}}\right.$ avec $\varepsilon_{\mathrm{p}}$ la déformation plastique par cycle, fig. 1), on a $\sigma_{\mathrm{m}} \alpha \varepsilon_{\mathrm{p}_{\mathrm{c}}}^{1 / 2}$. En admettant que les contraintes internes $\tau_{i}$ provenant des dislotions introduites contrôlent le durcissement, cela signifie que $\tau_{i} \alpha \varepsilon_{p_{c}}^{l / 2}$, soit avec $1 a$ relation classique $\tau_{i}{ }^{\alpha} \sqrt{\rho}, \rho$ étant la densité des dislocations, $\rho \alpha \varepsilon_{p_{c}} \cdot$ Cette évolution est proche de celle observée en déformation statique (12) et comparable aur résultats obtenus en fatigue sur le cuivre (6).

- La dépendance de la valeur de 1 'énergie dissipée avec le mode de sollicitation ( $\varepsilon_{\text {p }}$

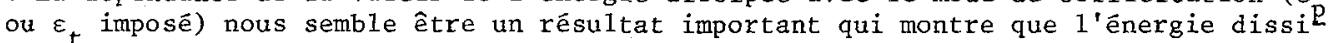
pée đépend du chemin parcouru en déformation plastique lorsque 1 'état microstructural caractérisé n'est pas stable. Ceci signifie que, lors des mesures de frottement intérieur usuelles, les résultats correspondent au cas où la déformation totale est imposée. Les différences observées selon le mode de sollicitation s'expliquent par la compétition entre deux phénomènes : augmentation de 1 'énergie dissipée due à la dêformation plastique et diminution de cette grandeur sous 1 'effet du durcissement qui en résulte.

\section{- Après déformation cyclique à saturation}

En ce qui concerne les résultats obtenus après fatigue, il apparẫt que des mécanismes différents doivent être pris en compte selon 1'amplitude de la déformation imposée $\varepsilon_{t}$, comme cela est le cas pour l'évolution du frottement intérieur après dêformation plastique monotone préalable (13), (14).

- A basse amplitude, $\varepsilon_{t}<10^{-4}$, il s'agit vraisemblablement de 1 'interaction dislocations-impuretés, comme en atteste la sensibilité au vieillissement (figure 6). Nos résultats ne font pas apparaître de pic de frottement intërieur, caractéristique de cette interaction, en raison probablement de la sensibilité insuffisante de notre appareillage aux đéformations où il devrait apparaître $\left(\varepsilon \simeq 10^{-5}\right)$. Pour expliquer une partie du durcissement après fatigue dans le cuivre, certains auteurs (6) ont suggéré qu'un mécanisme de traînage de crans pouvait intervenir. Ce mécanisme apparâ̂t presque exclusivement dans AI $6 \mathrm{~N}$ (15). Ceci nous conduit à penser que, dans nos expëriences, ce mécanisme intervient peu.

- Le fait qu'aux fortes amplitudes, le phẻnomène observé n'est pratiquement pas activé thermiquement (fig. 6) limite le nombre de mécanismes microscopiques susceptibles de le contrôler. Certains modèles de durcissement cyclique reposent sur la contrainte nécessaire aux basculements des dipoles ("flip flop" des dislocations). Ce mécanisme est activé thermiquement, mais I'énergie $W_{j}$ dépend de la distance $h$ entre les deux branches du dipole ( $W_{j} \alpha h$.ln h (16)). La valeur de h peut être telle que $W_{j}$ prenne des valeurs élevêes et qu'ainsi ce mécanisme apparaisse peu activé. En 1 absence de valeurs précises de $h$, il nous semble difficile de rejeter a priori ce mécanisme, même s'il apparaît peu probable.

Plus vraisemblable nous apparaît être 1 'existence des interactions du type dislocations-contraintes internes à grande distance. Ces contraintes peuvent être dues à la fois aux dislocations et aux boucles de dislocations créés par la fatigue. 
L'hypothèse des contraintes internes contrôlant la montée du frottement intêrieur de $10^{-4}$ à la saturation implique que le déplacement moyen des dislocations soit compatible avec la taille des cellules de dislocations observées. Si l'on admet que, pour la valeur de $2 \cdot 10^{-3}$, correspondant à I'état préfatigué initial, les dislocations atteignent les parois des cellules en se déplaçant sur une distance $\bar{\ell}: \simeq 1 \mu \mathrm{m}$, on trouve, avec la relation classique $\varepsilon=\zeta \rho \bar{\rho} \bar{l}(\zeta:$ facteur géométrique $\simeq 1 / 2)$, une densitê de dislocations $\rho \simeq 10^{9} \mathrm{~cm}^{-2}$. Cette valeur est en bon accord avec les densités de dislocations estimées à l'intérieur des canaux des structures fatiguées (6).

Nous pensons que 1 'on peút modêliser les évolutions observées de la manière sui-

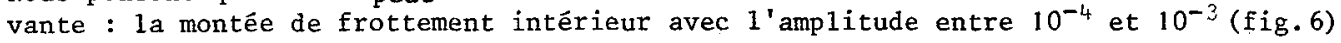
correspond à un déplacement moyen des dislocations à 1 'intérieur des cellules de plus en plus grand lorsque l'amplitude de déformation crồt. Pour une déformation $\varepsilon \simeq 10^{-3}$, les dislocations atteignent les parois des cellules; il y a alors interaction entre ces dislocations et celles des cellules correspondant vraisemblablement à des réarrangements et/ou à des annihilations de dislocations selon un mécanisme encore mal défini. Cet état correspond à l'état saturé initial. Le déplacement des dislocations étant probablement distribué, certaines d'entre elles atteignent les parois des cellules pour des amplitudes de déformation inférieures à $10^{-3}$.

Si 1 'on admet 1 'hypothèse de 1'annihilation, la densité des dislocations diminue et, par conséquent, le niveau de contrainte interne baisse. La mobilité des dislocations a ainsi tendance à croître, ce qui devrait conduire à une diminution de la contrainte maximale par cycle et à une augmentation du frottement intérieur. C'est effectivement ce qui a êté observé lorsque l'amplitude d'essai, après fatigue à saturation, atteint une valeur de $5 \cdot 10^{-4}$ bien inférieure à $2 \cdot 10^{-3}$, la déformation à saturation.

Enfin, 1'évolution différente de $\Delta$ et de $\sigma_{S}$ avec 1 'amplitude de déformation (fig. 5) dans le domaine des fortes valeurs conforte 1'idée émise (6) selon laquelle, au cours de la fatigue, il y a déformation des parois et de I'intérieur des cellules. En effet, aux fortes amplitudes de déformation, les déformations des parois nécessitent un accroissement de contrainte. Par contre, la forte densité de dislocations dans ces parois font qu'elles se déplacent peu, ce qui ne conduit pas à une augmentation sensible du niveau de l'énergie dissipée.

En conclusion, l'êtude de l'énergie dissipée a permis d'apporter un certain éclairage sur les mécanismes microscopiques de la fatigue de 1 'aluminium $5 \mathrm{~N}$. I1 reste que le rôle des différents types de contraintes internes doit être précisé : celles dues aux défauts eux-mêmes, ainsi que celles provenant de la compatibilité de la déformation à assurer entre les parois et 1 'intérieur des cellules de dislocation. Une étude plus approfondie des mécanismes microscopiques doit s'appuyer en outre sur des études complètes au MET et sur des résultats expérimentaux mettant en évidence 1 'effet de la température.

\section{REFERENCES}

(1) C.E. FELTNER - Phil. May., 12, pp. 1229-1248 (1965).

(2) J.E. PRATT - Acta Met., 15, February, pp. 315-327 (1967).

(3) J.M. FINNEY and C. LAIRD - Phil. May., 31, pp. 339-366 (1975).

(4) Z.S. BASINSKI, A.S. KORBEL and S.J. BASINSKI - Acta Met., vo1. 28, pp. 191-207 (1979).

(5) H. MUGHRABI - Materials Science and Engineering, 33, pp. 207-223 (1978).

(6) D. KUHLMANN-WILSDORF and C. LAIRD - Materials Science and Engineering, 37, pp. 111-120 (1979).

(7) J.C. FIGUEROA, S.P. BHAT, R. DE LA VEAUX, S. MURZENSKI and C. LAIRD - Acta Met., 29, pp. 1667-1678 (1981). 
(8) J.L. CHEVALIER - Thèse de Docteur-Ingénieur, Grenoble (1972) .

(9) B. TIRBONOD - Thèse $\mathrm{N}^{\circ} 298$, Ecole Polytechnique de Lausanne (1977).

(10) M. OMRI, J. CHICOIS, R, FOUGERES et A. VINCENT - ECIFUA 4, Lyon (1983).

(11) B.J. LAZAN - Damping of materials and members in structural mechanics, Pergamon Press (1968).

(12) J. CHICOIS, A. HAMEL, R. FOUGERES, C. ESNOUF, G. FANTOZZI and J. PEREZ - Journa 1 de Physique, Colloque $\mathrm{C5}$, supplément au $\mathrm{n}^{\circ} 10$, tome 42, pp. 169-173 (1981).

(13) P. PEGUIN, J. PEREZ and P. GOBIN - Transactions of the Metallurgica Society of AIME, 239, pp. 438-451 (1967).

(14) H. KRESSEL and N. BROWN - in "Dislocations Dynamics", by Rosenfield and al., Mc Graw-Hil1 (1968).

(15) L. NO, J. SANJUAN, C. ESNOUE, G. FANTOZZI et A. BERNALDE - ECIFUA4, I:yon 1983,

(16) G. FANTOZZI, W. BENOIT, C. ESNOUF et J. PEREZ - Annales de Physique, 4, pp. 7104 (1979). 